\title{
Lychee Associated Encephalopathy: Myth or Reality?
}

\section{ANKIT MANGLA ${ }^{*_{1}}$, KANDLA SHARMA², VASUDA BHAGAT ${ }^{3}$, MEHTAB SINGH BHAGAT ${ }^{4}$}

Once again, lychee fruit is flashing in the Indian news headlines after being criticized for association with fatal encephalopathy in Muzaffarpur region of Bihar. More than a 100 children in the district have lost their lives to this mysterious deadly outbreak. The research work behind the etiopathology of this lethal disease is minimal. There is a constant need of reporting these kind of unusual breakouts and analysing the data on basis of clinical and laboratory records. The health professionals will have to focus the target population, using preventive and curative measures, but this could be done only if substantial research is available for meta-analysis to reach a decision that could effectively and satisfactorily control this fatal and life-threatening public health issue.

KEYWORDS: Lychee, Encephalopathy, Encephalitis

\section{INTRODUCTION}

Lychee, also known as litchi is a member of the Litchi Genus of the soapberry family, Sapindaceae. It is endemic to the Fujian and Guangdong provinces of China, where cultivation is documented from around 1000 years back. China is the major producer of lychees, with India being second in line. A few other countries from Southeast Asia and South Africa also cultivate this tree. It is also a good source of vitamin $\mathrm{C}$ and $\mathrm{B}_{2}$ and minerals like copper and potassium. It also has anti-inflammatory, antioxidant, anti-cancer, anti-microbial, anti-diabetic, anti-obesity, liverprotective and immune-boosting properties. Being rich in proanthocyanidins and polyphenols, they may also help to prevent serious cardiovascular issues and cancers. Lychee seed extract helps reduce insulin resistance, and the polyphenol oligonol found in the lychee fruit may help to deal with diabetes-related vascular problems.

In recent years, lychee has gained more popularity because of its association with unexplained outbreaks of encephalopathy, appearing to affect only children in India, and northern Vietnam during the lychee harvest season from May to June. It is commonly known as "chamki bukhar" in India and "Ac Mong" encephalitis in Vietnam. There have been recurrent epidemics of hypoglycaemic encephalopathy in Muzaffarpur, the largest lychee fruit cultivation region in India. Most of the affected children in the past few years come from poor socioeconomic backgrounds of rural Muzaffarpur. In 2019, the death toll crossed 100 in a span of few weeks.

This deadly disease was first reported from Muzaffarpur itself in 1995, followed by large outbreaks nearly every year, manifesting in the summer season, mostly affecting young children, and with a reported mortality of upto $60 \%$. Most of the children who were affected by the concerned disease were reported being apparently well in the evening with an instantaneous onset of altered consciousness in the early hours next morning, accompanied by seizures and hypoglycemic state without any sign or symptoms that could indicate some kind of infection.

Although many researchers acknowledge the fact that the presentation of the subjects being affected is that of an encephalopathy rather like an encephalitis, viewpoints are quite different on the etiological factors. ${ }^{1}$ There have been many schools of thoughts including the heat stroke theory. The limitation of outbreak in the summer season, particularly in the months of April to July, has been interpreted as a suggestive of heat induced encephalopathy. No laboratory test has been specifically characterized to consider or rule out heat related disease. As the cases are limited to children, early morning onset and nonappearance of very high body temperature in the majority are the reasons eliminating heat as the cause. An investigator believed that the enormous 
humidity of Muzaffarpur with a temperature of more than 40 degrees in summers was causing life threatening heat stroke leading to encephalopathy accompanied by central nervous system dysfunction as there was a sudden drop in number of reported cases after start of the rainy season and drop in temperature. $^{2}$ This theory was not entertained broadly as the affected subjects showed early morning onset of symptoms, hyperpyrexia was inconsistent in the reported cases and the inhabitants were found to have a habit of drinking plenty of water. The other proposed reason was the use of pesticides during lychee cultivation. The most accepted one is the hypothesis of toxic origin suggesting the presence of lower analogue of hypoglycin A, namely methylenecyclopropyl-glycine (MCPG) in litchi seeds. This compound was not previously analysed in the ripe or unripe lychee fruit, but had been reported to result in hypoglycaemic conditions and derangement of fatty acid $\beta$-oxidation in liver cell mitochondria in experimental animals. ${ }^{5}$ These characteristics were correlated with the clinical features of early morning symptoms and signs of hypoglycaemia in these affected children further suggesting chances of metabolic encephalopathy rather than viral encephalitis.

The significant association of malnutrition in these children further supported this theory. ${ }^{1}$ The most common presenting symptoms reported in a study by Sahni GS are fever and convulsions, unconsciousness, decerebrate rigidity, tachycardia, tachypnea, absent splenomegaly and CSF with raised pressure. The reported age of affected children is between 3-7 years. The mechanism of toxicity of MCPG is related to the formation of MCPF-CoA, which in turn inhibits several dehydrogenases that are responsible for gluconeogenesis, causing depletion of glucose reserve in the body. ${ }^{5}$ Healthy and properly nourished children are not affected since their glycogen and glucose store in the liver is abundant to maintain normal glucose levels in blood and probably gluconeogenesis is not stimulated. ${ }^{3}$

In the year 2015, Das M et al. reported in a study that per gram litchi seeds contain 1.8 micrograms of MCPG, the ripe and semi-ripe pulp showed a value of 0.187 and 0.566 micrograms/gram MCPG respectively. ${ }^{6}$ In a case control study by Shrivastava A et al, Metabolites of hypoglycin A, MCPG, or both were detected in $66 \%$ of urine specimens from casepatients and $90 \%$ of case-patient specimens had abnormal plasma acylcarnitine values, consistent with serious disorganisation of fatty acid metabolism. ${ }^{7}$ Thus, the association of the disease with the lychee harvest became more logical.

For those who are affected or report some linked symptoms, $10 \%$ dextrose infusion in addition to anticonvulsant medications and supportive care are the treatment modalities of choice. $^{8}$ Systemic epidemiological research work needs to be conducted to describe in detail the etiological factors and mechanism, which are triggering the disease almost every year in the lychee cultivating regions. Meanwhile, health education measures on prevention of heat stroke may possibly contribute to a decrease in the number of affected children. No child should be allowed to sleep at night without eating a healthy meal and the parents need to abstain their children from eating litchis. Minimising litchi consumption among young children, ensuring adequate nutritional status in young children, and implementing rapid corrective measures for hypoglycaemia in children with suspected illness will help to prevent this disease and decrease the associated mortality rate. ${ }^{9}$ Global understanding and research on the adverse effects of methylenecyclopropyl-glycine on the neurological system will lead to rapid diagnosis and treatment of this mysterious epidemic of deadly encephalopathy.

\section{REFERENCES}

1. Yewale V. Misery of mystery of Muzaffarpur. Indian Pediatr. 2014;51:605-6.

2. Sahni GS. Recurring epidemics of acute encephalopathy in children in Muzaffarpur, Bihar. Indian Pediatr. 2012; 49:502-3.

3. John TJ, Das M. Acute encephalitis syndrome in children in Muzaffarpur: Hypothesis of toxic origin. Curr Sci. 2014;106:1184-5

4. Gray DO, Fowden L. Alpha(Methylenecyclopropyl)glycine from Litchi seeds. Biochem J. 1962;82 (3): 385-9.

5. Melde K, Buettner $\mathrm{H}$, Boschert W, Wolf HPO, Ghisla S. Mechanism of hypoglycaemic action of methylenecyclopropylglycine. Biochem J. 1989;259:921-924.

6. Das M, Athana S, Singh SP, Dixit S, Tripathi A, John TJ. Litchi fruit contains methylene cyclopropylglycine. Curr. Sci. India. 2015;109:2195-2197.

7. Shrivastava A, Kumar A, Thomas JD, Laserson KF, Bhushan G, Carter MD, et al. Association of acute toxic encephalopathy with litchi consumption in an outbreak in Muzaffarpur, India, 2014: a case-control study. Lancet Glob Health. 2017;5:e458-66. 
8. Shah A, John J. Recurrent outbreaks of hypoglycaemic encephalopathy in Muzaffarpur, Bihar. Current Science. 2014;107:570-1.
9. Renjen PN, Chaudhary DM, Kumar A. Litchi Toxicity: The Mystery Disease. International Journal of Scientific Research.2018; 7(12): 34-35

Source of support: Nil, Conflict of interest: None declared

Cite this article as:

Mangla A, Sharma K, Bhagat V, Bhagat MS. Lychee Associated Encephalopathy: Myth or Reality?. Int Healthc Res J. 2019;3(3):113-115. https://doi.org/10.26440/IHRJ/0303.06248

\section{AUTHOR AFFILIATIONS:}

1. MD (Paediatrics), Consultant SPS Hospital, Ludhiana, Punjab. (Corresponding Author)

2. MD (Paediatrics), Associate Consultant, Fortis Hospital, Jaipur.

3. PG Student, Department of Oral Medicine and Radiology, DJ College of Dental Sciences and Research, Modinagar, UP.

4. MBBS, Medical Officer, Government of Jammu and Kashmir.

Contact corresponding author at: amanglaıı@gmail.com 